\title{
L'impact de la connaissance des langues officielles, du niveau de littératie et du pays d'origine sur le risque de surqualification au travail des immigrants canadiens Impact of knowledge of official languages, level of literacy and country of origin on the risk of labour market over-qualification for immigrants to Canada
}

\section{Alain Bélanger et Samuel Vézina}

Volume 45, numéro 2, automne 2016

Démographie et main-d’oeuvre

URI : https://id.erudit.org/iderudit/1040393ar

DOI : https://doi.org/10.7202/1040393ar

Aller au sommaire du numéro

Éditeur(s)

Association des démographes du Québec

ISSN

1705-1495 (numérique)

Découvrir la revue

Citer cet article

Bélanger, A. \& Vézina, S. (2016). L'impact de la connaissance des langues officielles, du niveau de littératie et du pays d'origine sur le risque de surqualification au travail des immigrants canadiens. Cahiers québécois de démographie, 45(2), 145-166. https://doi.org/10.7202/1040393ar

\section{Résumé de l'article}

À l'instar du revenu ou du taux d'emploi, le taux de surqualification est un indicateur d'intégration des immigrants sur le marché du travail. Comparativement à la moyenne de l'OCDE, le taux de surqualification au Canada est élevé, mais l'écart entre les immigrants et les natifs est relativement faible.

L'objectif de cette recherche est de mesurer l'impact de la connaissance des langues officielles et des compétences en littératie sur le risque plus élevé pour les immigrants d'être surqualifié par rapport aux natifs. Les données canadiennes de l'enquête du Programme pour l'évaluation internationale des compétences des adultes (PEICA) de 2012 sont utilisées. Au total, l'échantillon retenu compte 13624 cas et représente les 14,5 millions de Canadiens âgés de 25 à 64 ans détenant un diplôme d'études secondaires ou plus qui étaient en emploi au cours de la semaine précédant l'enquête.

Les résultats montrent que l'usage des langues officielles et le niveau de littératie ont un impact significatif sur la propension des travailleurs canadiens à être surqualifiés. Le fait de parler une langue non officielle à la maison et le fait d'avoir un faible niveau de littératie sont tous les deux associés à une augmentation de $70 \%$ du risque d'être surqualifié. Bien qu'il existe une relation entre langue et littératie, ces deux types de compétences expliquent une part distincte du risque de surqualification des travailleurs canadiens. 


\title{
Cahiers québécois de démographie
}

Vol. 45, n² 2, automne 2016, p. 145-166

\section{L'impact de la connaissance des langues officielles, du niveau de littératie et du pays d'origine sur le risque de surqualification au travail des immigrants canadiens}

\author{
ALAIN BÉLANGER* ET SAMUEL VÉZINA
}

\begin{abstract}
RÉSUMÉ
À l'instar du revenu ou du taux d'emploi, le taux de surqualification est un indicateur d'intégration des immigrants sur le marché du travail. Comparativement à la moyenne de l'OCDE, le taux de surqualification au Canada est élevé, mais l'écart entre les immigrants et les natifs est relativement faible.

L'objectif de cette recherche est de mesurer l'impact de la connaissance des langues officielles et des compétences en littératie sur le risque plus élevé pour les immigrants d'être surqualifié par rapport aux natifs. Les données canadiennes de l'enquête du Programme pour l'évaluation internationale des compétences des adultes (PEICA) de 2012 sont utilisées. Au total, l'échantillon retenu compte 13624 cas et représente les 14,5 millions de Canadiens âgés de 25 à 64 ans détenant un diplôme d'études secondaires ou plus qui étaient en emploi au cours de la semaine précédant l'enquête.

Les résultats montrent que l'usage des langues officielles et le niveau de littératie ont un impact significatif sur la propension des travailleurs canadiens à être surqualifiés. Le fait de parler une langue non officielle à la maison et le fait d'avoir un faible niveau de littératie sont tous les deux associés à une augmentation de $70 \%$ du risque d'être surqualifié. Bien qu'il existe une relation entre langue et littératie, ces deux types de compétences expliquent une part distincte du risque de surqualification des travailleurs canadiens.
\end{abstract}

\footnotetext{
* Institut national de la recherche scientifique, Centre Urbanisation Culture Société, Montréal, Canada (alain.belanger@ucs.inrs.ca)

MENTION : Les analyses contenues dans ce texte ont été réalisées au Centre interuniversitaire québécois de statistiques sociales (CIQSS), membre du Réseau canadien des centres de données de recherche (RCCDR). Les activités du CIQSS sont rendues possibles grâce à l'appui financier du Conseil de recherche en sciences humaines (CRSH), des Instituts de recherche en santé du Canada (IRSC), de la Fondation canadienne pour l'innovation (FCI), de Statistique Canada, du Fonds de recherche du Québec - Société et culture (FRQSC), du Fonds de recherche du Québec - Santé (FRQS) ainsi que de l'ensemble des universités québécoises qui participent à leur financement. Les idées exprimées dans ce texte sont celles des auteurs et non celles des partenaires financiers.
} 


\begin{abstract}
Impact of knowledge of official languages, level of literacy and country of origin on the risk of labour market over-qualification for immigrants to Canada

In the same way as income and employment rates, the rate of over-qualification is an indicator of the integration of immigrants income into the labor market. In Canada the over-qualification rate is high in comparison to the OECD average; however, the gap between immigrants and the native-born is relatively small.

The aim of this research is to measure the impact of knowledge of official languages and of literacy skills on the risk of over-qualification for immigrants in comparison with the native-born. Canadian data from the 2012 Programme for the International Assessment of Adult Competencies (PIAAC) were used. In total, the sample included 13,624 cases and represents the 14.5 million Canadians aged 25 to 64 who hold a secondary education diploma or more and who were in employment during the week preceding the survey.

The results show that the use of official languages and the level of literacy have a significant impact on the propensity of Canadian workers to be over-qualified. Not speaking an official language at home and having a low level of literacy are both associated with an increase of $70 \%$ in the risk of being over-qualified. Although there is a relationship between language and literacy, these two types of skill explain distinct parts of the risk of over-qualification among Canadian workers.
\end{abstract}

\title{
INTRODUCTION
}

T e Canada est depuis toujours un pays d'immigration. Cet aspect de l'histoire démographique canadienne s'avère particulièrement contemporain puisque le taux annuel d'immigration se maintient depuis les années 1990 à des niveaux pratiquement deux fois plus élevés que ceux observés aux États-Unis ou au Royaume-Uni. Les estimations officielles au $1^{\text {er }}$ juillet 2016 montrent un nombre record d'immigrants reçus au pays en 2015-2016, soit 320932 personnes, du jamais vu en plus de cent ans (Statistique Canada, 2016). En conséquence, la population née à l'étranger est en constante progression et atteignait $21 \%$ de la population totale en 2011, la plus forte proportion parmi les pays du G8 (Statistique Canada, 2013b). Selon Statistique Canada, cette proportion devrait continuer de croître pour représenter entre $25 \%$ et $28 \%$ de la population en 2031 (Caron Malenfant et collab. 2010).

La politique d'immigration canadienne est sélective, mais non discriminatoire. Elle est sélective puisque près des deux tiers des candidats sont admis selon une grille de points pour différents critères, la plupart reliés au capital humain des individus. En effet, cette grille accorde des points pour des caractéristiques telles que l'âge, la connaissance des langues officielles et le niveau d'éducation (Citoyenneté et immigration Canada, 2015). Il n'est donc pas étonnant de constater que les Canadiens nés à l'étranger sont beaucoup plus susceptibles de détenir un diplôme universitaire que les Canadiens nés au pays. Plus précisément, au recensement de 2011, plus du tiers (36\%) des immigrants âgés entre 25 et 64 ans détenaient un diplôme universitaire alors que cette proportion n'était que de $22 \%$ chez les Canadiens de naissance (Statistique Canada, 2013a), et ce, malgré la hausse des taux de diplomation pour les cohortes plus récentes (Statistique Canada, 2013c). L'orientation non discriminatoire de la politique se traduit quant à elle par une diversification notable de l'origine des immigrants. Cela entraine certains changements dans la composition linguistique des Canadiens nés à l'étranger, et ce, bien que la grille de sélection privilégie les immigrants connaissant une des deux langues officielles du Canada. En 1981, 10 \% des Canadiens étaient 
allophones ${ }^{1}$, alors que cette proportion atteignait $21 \%$ en 2011 . On s'attend à ce que ce pourcentage grimpe à $30 \%$ en 2031 (Caron Malenfant et collab. 2010).

Malgré le fait que les immigrants soient en moyenne plus scolarisés que les natifs, de nombreuses études ont montré que l'intégration économique des immigrants n'est pas instantanée. Les immigrants admis au Canada depuis 1990, voire depuis 1980, connaissent des difficultés plus grandes à s'insérer sur le marché de l'emploi par rapport aux cohortes plus anciennes d'immigrants (Aydemir et Skuterud, 2004; Frenette et Morissette, 2005; Li, 2003; Picot et Hou, 2009; Skuterud, 2011). Cette plus grande difficulté d'insertion se constate sur toutes les mesures de l'intégration économique, que ce soit le niveau d'activité, le taux de chômage, le revenu ou la propension à être surqualifié pour l'emploi occupé (OCDE, 2012).

Plusieurs hypothèses ont été avancées pour expliquer cette tendance. La durée de résidence au pays, la moins bonne maîtrise des langues officielles, la transférabilité difficile des connaissances et de l'expérience acquises à l'étranger sur le marché du travail canadien, la non-reconnaissance des diplômes acquis à l'étranger par les employeurs canadiens et la discrimination subie par les nouveaux arrivants provenant majoritairement de pays non occidentaux sont des éléments généralement mis de l'avant pour expliquer le phénomène (Causa et Jean, 2007; Green et Worswick, 2012; Hou et Picot, 2014; Picot et collab. 2008; Schaafsma et Sweetman, 2001; Sweetman, 2004, 2014 ;). Les études antérieures ont montré que les difficultés d'intégration des immigrants pouvaient effectivement s'expliquer par la durée de résidence (Chiswick, 1978 ; Lubotsky, 2007), l'âge à l'arrivée (Portes et Zhou, 1993; Schaafsma et Sweetman, 2001), le changement dans les pays sources et la possible discrimination qui en aurait découlé (Hum et Simpson, 2000 ; Pendakur et Pendakur, 1998, 2002) et un changement dans le capital humain des immigrants (Chiswick et Miller, 2002, 2009a ; Ferrer et Riddell, 2004; Sweetman et McBride, 2004).

Plus rarement, le niveau des compétences des immigrants est mis en cause. Pourtant, des auteurs ont récemment montré que les différences des compétences en traitement de l'information telles que mesurées par le score en littératie ${ }^{2}$ expliquent une grande partie des écarts de revenu des immigrants par rapport aux natifs. Ferrer et ses collaborateurs (2006) s'inscrivent à l'encontre des explications communes mettant de l'avant des raisons liées à la discrimination pour expliquer les différences observées entre le revenu des immigrants et celui des natifs. À l'aide des données de l'Enquête sur la littératie et la compétence des adultes (ELCA) de 1994, ils montrent que les immigrants et les natifs obtiennent des rendements similaires pour leur compétence en littératie sur le marché de l'emploi et que chez les diplômés universitaires, les scores de littératie plus élevés des natifs expliqueraient environ les deux tiers des écarts de revenus observés entre immigrants et Canadiens de naissance. Bonikowska et ses collaborateurs (2008), utilisant les données canadiennes de l'Enquête internationale sur l'alphabétisation et les compétences des adultes (EIACA) de 2003, démontrent également le lien fort entre les compétences et l'intégration économique. Les auteurs montrent que si on arrivait à élever le niveau moyen de littératie des immigrants mascu-

1. Au Canada, on définit les allophones comme étant des personnes dont la langue maternelle n'est ni le français ni l'anglais.

2. La littératie est définie comme la capacité de comprendre, d'évaluer, d'utiliser et de s'approprier des textes écrits (Statistique Canada 2013d). 
lins détenant un diplôme d'études secondaires (ou plus) de façon à ce qu'il atteigne celui des Canadiens de naissance, on éliminerait complètement l'écart de revenu entre les deux groupes. Chez les femmes, les immigrantes seraient avantagées par rapport à leurs homologues nées au pays.

Le principal objectif de cet article est de mesurer l'effet des compétences linguistiques et du niveau de littératie sur la différence de risque pour les immigrants d'être surqualifié par rapport aux natifs. L'hypothèse veut que les différences de compétences entre les deux groupes de population expliquent une partie significative de la surqualification accrue des immigrants. La présente étude bonifie et ajoute aux connaissances concernant l'intégration économique des immigrants au Canada en mesurant l'impact du niveau de littératie et des compétences linguistiques sur le risque d'être surqualifié dans l'emploi occupé. D’une part, les données de l'enquête de 2012 du Programme pour l'évaluation internationale des compétences des adultes (PEICA), plus récentes que l'ELCA et que l'EIACA, sont utilisées pour mesurer la relation entre le niveau de littératie des deux populations et l'intégration économique des immigrants. D'autre part, plutôt que l'écart de revenu entre les immigrants et les natifs, cet article utilise le risque d'être surqualifié pour l'emploi occupé comme une autre mesure de l'intégration économique.

L'article se divise en quatre autres sections. La première section présente une revue des résultats des études antérieures portant sur le lien entre le risque d'être surqualifié et le statut d'immigrant. Elle est suivie d'une description de la population ciblée par l'enquête utilisée et des variables dépendantes et indépendantes du modèle de régression logistique. Les résultats de l'analyse multivariée sont ensuite présentés, suivis d'une discussion des implications des principaux résultats de recherche.

\section{SURQUALIFICATION ET STATUT D'IMMIGRATION: UNE REVUE DE LA LITTÉRATURE}

Succinctement, la surqualification est un concept qui réfère à l'inadéquation entre les compétences de l'employé et celles exigées par l'emploi qu'il occupe. La surqualification se définit généralement en fonction du niveau d'éducation. Un travailleur est surqualifié si ses compétences ${ }^{3}$ sont plus élevées que celles exigées pour son emploi. Il existe différentes façons de mesurer la surqualification : l'approche subjective par autoévaluation, l'approche statistique et l'approche objective ${ }^{4}$.

3. Ou, plus généralement, son niveau d'éducation.

4. Voir Boudarbat et Montmarquette (2013) et Roy (2014) pour une description détaillée des méthodes de mesure de la surqualification. En bref, l'approche subjective par autoévaluation directe ou indirecte est basée sur les réponses données à un questionnaire et peut être soumise à plusieurs types de biais. L'approche statistique compare le niveau d'éducation de l'employé avec celui de la moyenne des personnes ayant la même profession et considère qu'un employé est surqualifié s'il a significativement plus d'années de scolarité que la moyenne des personnes employées dans sa profession. Quant à l'approche objective, elle permet d'établir la surqualification en comparant le niveau de scolarité de l'employé avec celui requis pour l'emploi qu'il occupe. Elle nécessite une classification établie par des experts de l'analyse des professions. Le taux de surqualification varie selon l'approche retenue pour le calcul. Selon Hartog (2000), l'approche objective est préférable, bien qu'elle ne soit pas sans limites, notamment en ce qui concerne l'actualisation de la grille de comparaison. 
L'approche objective est retenue dans le cadre de cet article, à l'instar d'autres travaux scientifiques importants dans le domaine (Boudarbat et Montmarquette, 2013; Chiswick et Miller, 2009a; Dumont et Monso, 2007; Galarneau et Morissette, 2004, 2008; Green et collab. 2007).

Les études récentes sur la surqualification ont permis de montrer, d'une part, que les travailleurs surqualifiés ont des revenus significativement plus bas que les travailleurs non surqualifiés et, d'autre part, que certaines franges de la population sont plus à risque d'être surqualifiés, notamment les immigrants (Causa et Jean, 2007). Quelques études ont tenté d'identifier les déterminants de la surqualification dans le contexte d'une intégration économique plus difficile des immigrants. S’intéressant à quelques pays européens et à l'Australie, Dumont et Monso (2007) montrent l'importance des compétences en littératie et des caractéristiques individuelles pour expliquer la surqualification accrue des immigrants. En utilisant les données de l'ECLA, ils estiment que les compétences en littératie permettent d'expliquer un tiers de la surexposition des immigrants à la surqualification. La série de modèles logit élaborés par les auteurs révèle par ailleurs l'importance de tenir compte du pays d'obtention du plus haut diplôme et des compétences linguistiques des individus. Lorsque l'effet de ces variables est neutralisé, on ne mesure plus d'écart significatif entre les natifs et les immigrants quant à leur probabilité d'être surqualifié.

Du côté des études canadiennes, deux recherches récentes sur la surqualification des nouveaux diplômés universitaires montrent l'importance des divers déterminants recensés dans la littérature : l'âge, le sexe, le niveau d'éducation et le lieu d'obtention du plus haut diplôme, le domaine d'étude, le type de région et la province de résidence (Uppal et LaRochelle-Côté, 2014). Utilisant les données de l'Enquête nationale auprès des ménages de 2011, les auteurs montrent que le statut d'immigrant demeure significatif pour expliquer le risque de surqualification, y compris les immigrants diplômés d'un établissement canadien ou américain. Cependant, l'effet des compétences linguistiques et de niveau de littératie n'est pas pris en compte dans l'analyse.

Avec un modèle d'analyse semblable, l'étude de Ledent et ses collaborateurs (2014) analyse le phénomène en se concentrant sur les immigrants qui résident sur l'île de Montréal. Grâce aux données du recensement de 2006, les compétences linguistiques sont intégrées au modèle d'analyse, en plus d'autres caractéristiques telles que l'appartenance à une minorité visible. Les auteurs montrent qu'à caractéristiques égales, les immigrants ne sont pas plus surqualifiés que les natifs. On observe aussi un lien fort entre la maîtrise des langues officielles et le risque d'être surqualifié. Finalement, on note un impact important du statut de minorité visible pour certains groupes, plus particulièrement pour les noirs et les Philippins.

Plus récemment, l'étude de Bérard-Chagnon (2015) a analysé la surqualification des travailleurs en Ontario avec les données du PEICA. On démontre que les individus avec un faible niveau de littératie sont significativement plus à risque d'être surqualifiés que ceux avec un fort niveau de littératie. Le modèle d'analyse prend en compte le statut d'immigrant, mais l'impact de cette variable de contrôle n'est ni divulgué ni commenté.

Notons finalement l'étude de LaRochelle-Côté et Hango (2016) qui utilise également les données du PEICA pour étudier le phénomène de surqualification chez les travailleurs de 25 à 64 ans détenant un diplôme universitaire. Contrairement à notre analyse, leur modèle n'inclut pas les compétences en littératie comme facteur explicatif possible de la surqualification. Ils montrent néanmoins que l'impact du statut d'immigrant sur la surqualification n'est négatif que pour les 
immigrants ayant obtenu leur diplôme à l'étranger. Plus exactement, on voit que les immigrants diplômés au Canada sont en fait deux fois moins à risque d'être surqualifiés que les natifs.

\section{DONNÉES ET MÉTHODE}

PEICA est une enquête internationale menée dans plusieurs pays sous la direction de l'OCDE. Le volet canadien de l'enquête utilisé ici compte 27054 répondants et, une fois pondéré, l'échantillon est représentatif de la population canadienne âgée de 16 à 65 ans, excluant les militaires, les individus vivant en institution, les autochtones habitant sur des réserves et les résidents de certaines régions peu peuplées. La population immigrante est suréchantillonnée, ce qui permet de conduire des analyses avec une force statistique intéressante.

Dans cet article, l'analyse est, par définition, limitée aux répondants en emploi. On retranche donc les chômeurs et les non-actifs (étudiants, retraités, stagiaires, invalides, femmes ou hommes à la maison) de l'échantillon. De plus, étant donné que notre question de recherche porte sur l'intégration économique, et afin de maximiser l'homogénéité de notre échantillon, nous retirons aussi les personnes âgées de moins de 25 ans dont le parcours scolaire est susceptible d'être inachevé. Les répondants âgés de 65 ans sont également omis de nos analyses puisqu'ils sont admissibles à une prestation de retraite. Nous retirons aussi les répondants qui ne détiennent pas au moins un diplôme d'études secondaires, car ceux-ci sont soumis à un risque nul d'être surqualifié. Au total, l'échantillon retenu compte 13624 individus représentatifs de la population canadienne en emploi âgée entre 25 et 64 ans et diplômée du secondaire ou plus. Nous utilisons la pondération adéquate pour que nos estimations tiennent compte du plan d'échantillonnage complexe de l'enquête (poids jackknife) et des procédures de régressions logistiques (piaacreg) développées spécifiquement pour l'architecture de la base de données du PEICA (voir Pokropek et Jakubowski (2014)).

\section{DESCRIPTION DES VARIABLES}

\section{La variable dépendante}

La surqualification est définie en comparant le niveau d'études des individus au niveau de compétence requis par le poste occupé. Ce niveau de compétence normalement requis par l'emploi est défini par les analystes du ministère fédéral Emploi et Développement social Canada (EDSC) à l'aide de la Classification nationale des professions (CNP). Plus précisément, les emplois sont classés sous cinq grands niveaux de compétences:

- Niveau o : emplois en gestion,

- Niveau A : emplois qui requièrent une formation universitaire,

- Niveau B : emplois qui requièrent une formation collégiale ou un programme technique,

- Niveau C : emplois qui requièrent une formation de niveau secondaire, et

- Niveau D : emplois caractérisés par une formation en cours d'emploi (moins qu'un niveau secondaire). 
Un travailleur est surqualifié lorsque le niveau d'éducation du répondant est supérieur à celui de l'emploi qu'il occupe. Par exemple, un répondant détenant un diplôme universitaire qui occupe un emploi de niveau B, C ou D est considéré comme étant surqualifié. Par définition, les personnes n'ayant aucun diplôme ne peuvent connaitre la surqualification, tout comme les personnes occupant un poste de gestion.

\section{Les variables indépendantes}

\section{Compétences linguistiques}

Les enquêtes canadiennes contiennent généralement plusieurs questions linguistiques permettant au répondant de déclarer sa langue maternelle, sa langue d'usage, sa connaissance des langues officielles ou sa langue au travail. L'enquête PEICA contient des questions sur la langue maternelle et la langue d'usage à la maison qui permettent de créer une variable dérivée à trois catégories servant de proxy aux compétences linguistiques. Idéalement, une mesure objective des compétences linguistiques serait nécessaire pour mesurer tout l'impact de cette dimension sur l'intégration économique des immigrants. Malheureusement, une telle mesure n'existe dans aucune enquête canadienne (Corbeil, 2011), même si la langue est une caractéristique historiquement et socialement importante de la diversité canadienne (Porter, 1965).

Dans notre échantillon, les Canadiens de langue maternelle officielle (anglais ou français) sont distingués des allophones, qui sont eux-mêmes divisés en deux groupes : ceux qui utilisent une langue officielle à la maison et ceux qui utilisent une autre langue à la maison. Sabourin et Bélanger (2014) ont montré que les allophones qui utilisent une des deux langues officielles à la maison sont mieux rémunérés que ceux qui utilisent une langue tierce. Ils avancent l'hypothèse que les allophones qui parlent le français ou l'anglais à la maison développent, à l'usage, de meilleures compétences linguistiques rémunératrices sur le marché du travail canadien. L'idée testée ici est que ces meilleures compétences réduiraient, voire élimineraient, les différences de surqualification par rapport aux Canadiens de langue officielle qui forment la catégorie de référence dans le modèle 5 .

\section{Compétences en littératie}

Les compétences en littératie d'un individu se réfèrent à sa capacité à « comprendre, évaluer, utiliser et à s'approprier des textes écrits pour participer à la société, réaliser ses objectifs et développer ses connaissances et son potentiel»(OCDE, 2014). Dans l'enquête PEICA, les compétences

5. Il a été suggéré par un évaluateur d'utiliser les réponses à la question posée dans l'enquête aux allophones leur permettant d'autoévaluer leur capacité de parler le français et l'anglais. Nous préférons notre variable linguistique, car l'autoévaluation est subjective et instable. En effet, chez près d'un tiers des répondants de langue tierce à l'Enquête longitudinale sur les immigrants au Canada, la capacité autoévaluée diminue entre deux passages. Le répondant déclare des capacités élevées quelque temps après son arrivée au Canada, mais tend à évaluer plus faiblement ses capacités linguistiques à mesure que sa durée de résidence au Canada augmente. Nous croyons aussi que notre variable est mieux adaptée aux recommandations de politiques. Finalement, elle a aussi l'avantage d'être utilisée dans plusieurs autres enquêtes canadiennes et dans les recensements. 
en littératie sont définies et mesurées en termes fonctionnels, en ce sens que les items qui servent à la mesurer sont présentés dans le contexte de tâches à exécuter dans le cadre de la vie quotidienne. Les compétences en littératie sont mesurées directement par le biais de tâches de traitement de l'information de complexité croissante à compléter par les répondants. Il résulte du traitement des résultats des répondants un ensemble de dix valeurs plausibles qui correspondent à un score de compétence sur une échelle continue allant de o à 500 points. Dans le but d'en faciliter l'analyse, les scores sont regroupés en cinq niveaux de compétence. Les niveaux 1 et 2, scores de o à 225 et de 226 à 275 respectivement, correspondent à des compétences faibles et considérées comme insuffisantes pour fonctionner aisément dans la société actuelle. Le niveau 3, scores allant de 276 à 325, est considéré comme le niveau minimal souhaité de compétence pour bien fonctionner (Statistique Canada et OCDE, 2005), alors que les niveaux 4 (326 à 375 points) et 5 (376 points et plus) témoignent de compétences élevées. Le rapport du PEICA révèle que $51 \%$ de la population du Canada peut exécuter avec succès des tâches de niveau 3 ou plus (Statistique Canada 2013d). Ainsi, $49 \%$ des Canadiens obtiennent un score inférieur à 276 points (niveau faible). Dans notre analyse, nous avons choisi de dichotomiser notre mesure des compétences en littératie pour distinguer les répondants qui se situent au niveau 3 ou plus de ceux qui se trouvent en dessous de ce seuil.

\section{La variable d'intérêt analytique: le statut d'immigrant}

Étant donné l'objectif de cette recherche, il convient de prendre en compte le statut d'immigrant dans les analyses. Sur la base de la revue de la littérature, nous savons que l'effet de cette dimension varie selon les caractéristiques propres aux immigrants. Par exemple, les immigrants récemment arrivés au pays sont soumis à un risque plus élevé de connaitre la surqualification ceteris paribus. Il en va de même pour le pays d'origine, l'âge à l'arrivée et le pays d'obtention du plus haut diplôme. Puisque toutes ces caractéristiques ne s'appliquent essentiellement qu'aux immigrants, insérer séparément chacune de ces caractéristiques crée une colinéarité quasi parfaite entre elles et avec le statut d'immigrant. Pour tenir compte de tous ces effets possiblement contradictoires, nous avons recours à une variable composite du statut d'immigrant. Cette variable est dérivée à l'aide de plusieurs questions de l'enquête : période d'immigration, âge à l'arrivée, pays de naissance et pays d'obtention du plus haut diplôme. Cela nous permet d'aller au-delà des études recensées plus haut et de tenir compte de caractéristiques pertinentes pour l'étude de la surqualification.

Pour créer une variable dont les catégories sont mutuellement exclusives, nous devons accorder préséance à l'une ou l'autre des caractéristiques contenues dans la variable composite. La variable composite est donc construite en tenant compte, dans l'ordre, de la période d'immigration, de l'âge à l'arrivée, de l'origine et finalement du lieu d'obtention du plus haut diplôme. Ainsi, la première catégorie (immigrants récents) regroupe tous les immigrants admis depuis cinq ans ou moins, peu importe leurs autres caractéristiques. La seconde (génération 1,5) regroupe tous les immigrants arrivés avant l'âge de 15 ans à l'exception de ceux admis durant les cinq années précédant l'enquête. Les huit autres catégories désagrègent les immigrants non récents et admis après l'âge de 15 ans selon leur pays de naissance. Elles permettent de distinguer les immigrants nés dans un pays développé pour qui les difficultés d'intégration sont moindres des autres. Compte tenu 
des faibles effectifs, il est impossible de distinguer chacun des pays de naissance et des regroupements ont donc été faits. Une catégorie isole les immigrants originaires des Philippines, car la majorité de ceux-ci sont admis au Canada par le biais du Programme d'aide familial résidant qui a un effet spécifique sur la surqualification (Ledent et collab. 2014). À partir des grandes régions de

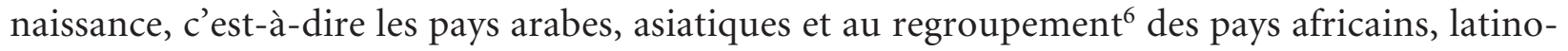
Américains et autres, six autres catégories sont formées en séparant chacun de ces trois groupes selon le lieu d'obtention du plus haut diplôme (pays développé ${ }^{7}$ ou non). Cette distinction selon le lieu d'obtention du plus haut diplôme est pertinente puisqu'elle permet de tenir compte de la plus grande transférabilité des diplômes occidentaux sur le marché du travail canadien (Bonikowska et collab. 2010 ; Chiswick et Miller, 2009b ; Sweetman, 2014).

\section{Les variables de contrôle}

Les modèles de régression contrôlent aussi pour le sexe, le groupe d'âge, le lieu de résidence et le domaine d'étude. Les répondants sont regroupés dans quatre groupes d'âge décennaux allant de 25 à 34 ans, de 35 à 44 ans, de 45 à 54 ans et de 55 à 64 ans. En ce qui concerne le lieu de résidence, nous avons isolé dans des catégories distinctes les trois grandes villes canadiennes Toronto, Montréal et Vancouver, où vivent la grande majorité des immigrants du Canada. Deux autres catégories ont été créées pour regrouper, d'une part, tous les répondants vivant en ville ("Autres RMR/ $\left.\mathrm{AR}^{8} »\right)$, laissant les répondants vivant en milieu rural dans la seconde catégorie "Ailleurs au Canada ». Finalement, sur la base de la revue de la littérature, nous avons réduit à trois catégories le nombre de domaines d'études. Sachant que les diplômés de sciences sociales et sciences humaines sont plus à risque d'être surqualifiés (Uppal et LaRochelle-Côté, 2014; LaRochelle-Côté et Hango, 2016), nous les avons regroupés dans la catégorie « Domaines les plus à risque de surqualification ». Dans la catégorie "Domaines les moins à risque de surqualification ", nous avons regroupé les diplômés de sciences et génie, des sciences informatiques ainsi que des sciences de l'éducation et de la santé. Dans une troisième catégorie résiduelle "Autres », nous avons isolé les répondants dont le domaine d'études est inconnu, qui n’est pas spécifié ( Études générales ») ou qui correspond à des emplois de «Services », lesquels peuvent se retrouver d'un bout à l'autre du spectre du risque de surqualification.

6. Les immigrants originaires de l'Afrique sub-saharienne, de l'Amérique latine et de l'Océanie ont dû être regroupés dans la même catégorie par manque d'effectifs suffisants de répondants pour conduire des analyses plus détaillées.

7. Les pays développés correspondent aux pays de l'Europe du Nord et de l'Ouest, aux pays d'Amérique du Nord ainsi qu'à l'Australie, la Corée du Sud, le Japon, la Nouvelle-Zélande et Singapour.

8. Les expressions techniques "région métropolitaine de recensement» (RMR) et «agglomération de recensement» (AR) sont utilisées par Statistique Canada pour désigner les plus grandes villes du pays. Une RMR doit avoir une population d'au moins 100 ooo habitants avec un centre urbain (communément appelé "noyau ») comptant au moins 50 ooo habitants. L'AR doit avoir un noyau au moins 10 ooo habitants (Statistique Canada, 2015). 


\section{DESCRIPTION DE LA POPULATION À L'ÉTUDE}

Au total, l'échantillon retenu compte 13624 cas et représente les 12,4 millions de Canadiens âgés de 25 à 64 ans détenant un diplôme d'études secondaires ou plus et qui étaient en emploi au cours de la semaine précédant l'enquête. Le Tableau 1 présente la répartition de la population à l'étude selon les différentes caractéristiques retenues pour l'analyse statistique. Globalement, un peu plus du quart $(27,6 \%)$ de la population des travailleurs âgés de 25 à 64 ans occupe un emploi pour lequel il est surqualifié. Le taux de surqualification est nettement plus élevé chez les immigrants $(38,9 \%)$ que chez les natifs $(24,0 \%)$.

\section{TABLEAU 1}

Distribution (en \%) de la population en emploi âgée de 25 à 64 ans détenant au moins un diplôme d'études secondaires, suivant diverses variables, par statut d'immigration

\begin{tabular}{|c|c|c|c|c|}
\hline \multirow{2}{*}{ Variables } & & \multirow{2}{*}{$\begin{array}{l}\text { Population } \\
\text { totale }\end{array}$} & \multicolumn{2}{|c|}{ Statut d'immigration } \\
\hline & & & Natifs & Immigrants \\
\hline \multicolumn{5}{|c|}{ Variable dépendante } \\
\hline \multirow{2}{*}{$\begin{array}{l}\text { Occupe } \\
\text { un emploi } \\
\text { surqualifié }\end{array}$} & Non & 72,4 & 76,0 & 61,1 \\
\hline & Oui & 27,6 & 24,0 & 38,9 \\
\hline \multicolumn{5}{|c|}{ Variable d'intérêt analytique } \\
\hline \multirow{12}{*}{$\begin{array}{l}\text { Variable } \\
\text { composite } \\
\text { des } \\
\text { caractéristiques } \\
\text { des immigrants }\end{array}$} & Natifs & 75,7 & 100,0 & 0,0 \\
\hline & Imm. récents & 1,8 & 0,0 & 7,2 \\
\hline & Imm. arrivés avant l'âge de 15 ans & 5,3 & 0,0 & 22,0 \\
\hline & Imm. nés dans un pays développé & 2,2 & 0,0 & 9,0 \\
\hline & Imm. nés en Europe de l’Est/Europe centrale & 2,2 & 0,0 & 9,2 \\
\hline & Imm. nés aux Philippines & 1,8 & 0,0 & 7,4 \\
\hline & $\begin{array}{l}\text { Imm. nés dans un pays arabe et diplômés d'un } \\
\text { pays développé }\end{array}$ & 0,4 & 0,0 & 1,8 \\
\hline & $\begin{array}{l}\text { Imm. nés dans un pays arabe et diplômés d'un } \\
\text { autre pays }\end{array}$ & 0,6 & 0,0 & 2,4 \\
\hline & Imm. nés en Asie et diplômés d'un pays développé & 2,2 & 0,0 & 9,2 \\
\hline & Imm. nés en Asie et diplômés d'un autre pays & 3,8 & 0,0 & 15,7 \\
\hline & $\begin{array}{l}\text { Imm. nés ailleurs (Afr., Am. lat., Océanie) et } \\
\text { diplômés d'un pays développé }\end{array}$ & 2,0 & 0,0 & 8,2 \\
\hline & $\begin{array}{l}\text { Imm. nés ailleurs (Afr., Am. lat., Océanie) et } \\
\text { diplômés d'un autre pays }\end{array}$ & 1,9 & 0,0 & 7,8 \\
\hline \multicolumn{5}{|c|}{ Variables de contrôle } \\
\hline \multirow{2}{*}{ Sexe } & Hommes & 52,8 & 51,9 & 55,4 \\
\hline & Femmes & 47,2 & 48,1 & 44,6 \\
\hline
\end{tabular}


TABLEAU 1 (suite)

\begin{tabular}{|c|c|c|c|c|}
\hline \multirow{4}{*}{ Groupe d'âge } & $25-34$ ans & 25,2 & 26,6 & 21,0 \\
\hline & $35-44$ ans & 27,0 & 25,9 & 30,3 \\
\hline & $45-54$ ans & 30,2 & 30,4 & 29,6 \\
\hline & $55-64$ ans & 17,6 & 17,1 & 19,1 \\
\hline \multirow{6}{*}{$\begin{array}{l}\text { Lieu } \\
\text { de résidence }\end{array}$} & Toronto & 17,3 & 10,2 & 39,7 \\
\hline & Montréal & 11,4 & 11,0 & 12,7 \\
\hline & Vancouver & 7,4 & 5,5 & 13,2 \\
\hline & Autres RMR/AR & 46,3 & 51,2 & 31,0 \\
\hline & Ailleurs au Canada & 16,5 & 20,7 & 3,3 \\
\hline & Inconnu & 1,1 & 1,5 & 0,1 \\
\hline \multirow{3}{*}{$\begin{array}{l}\text { Domaine } \\
\text { d'études }\end{array}$} & Domaines les moins à risque de surqualification & 43,3 & 42,0 & 47,2 \\
\hline & Domaines les plus à risque de surqualification & 27,5 & 26,5 & 30,5 \\
\hline & Autres (Études générales, Services, Inconnu) & 29,2 & 31,5 & 22,3 \\
\hline \multicolumn{5}{|c|}{ Variables reliées au capital humain } \\
\hline \multirow{3}{*}{$\begin{array}{l}\text { Connaissance et } \\
\text { usage } \\
\text { des langues } \\
\text { officielles }\end{array}$} & Langue maternelle (LM) officielle & 76,1 & 94,4 & 18,9 \\
\hline & LM non off./Langue d'usage officielle & 11,1 & 4,7 & 31,1 \\
\hline & LM non off./Langue d'usage non off. & 12,9 & 1,0 & 50,0 \\
\hline \multirow{2}{*}{$\begin{array}{l}\text { Niveau } \\
\text { de littératie }\end{array}$} & Inférieur au niveau 3 & 40,7 & 35,6 & 56,8 \\
\hline & Équivalent ou supérieur au niveau 3 & 59,3 & 64,4 & 43,2 \\
\hline N pondéré & & 12427300 & 9410500 & 3016800 \\
\hline \multicolumn{2}{|c|}{ Pourcentage pondéré } & 100,0 & 75,7 & 24,3 \\
\hline
\end{tabular}

Source: PEICA 2012.

Un peu plus des trois quarts de l'échantillon est formé de Canadiens de naissance. Parmi le quart restant, $7 \%$ sont des immigrants récents (admis depuis cinq ans ou moins) et un peu plus de $20 \%$ sont des immigrants admis au Canada avant l'âge de 15 ans (génération 1,5), tous pays d'origine ou de diplomation confondus. Quelque $18 \%$ des immigrants sont nés dans un pays développé ou en Europe de l'Est ou du centre, répartis en parts égales entre les deux catégories. Un peu plus de la moitié des immigrants (hormis les immigrants récents ou arrivés avant 15 ans) sont originaires de pays autres. Ils sont répartis en sept catégories. Les immigrants originaires d'Asie sont les plus nombreux composant le quart des immigrants de la population à l'étude, auxquels il faut ajouter $7 \%$ de Philippins. Près des deux tiers des Asiatiques non philippins ont obtenu leur plus haut diplôme dans un pays non développé. Les Arabes ne représentent qu'environ $5 \%$ de l'échantillon et un peu moins de $60 \%$ d'entre eux ont obtenu leur plus haut diplôme dans un pays non développé. Finalement, les immigrants d'origine africaine, latino-américaine ou autre représentent un peu plus de $15 \%$ de la population à l'étude et on retrouve parmi eux autant de diplômés d’un pays développé que non développé. 
Quant aux variables de contrôle, l'échantillon compte légèrement plus d'hommes que de femmes, mais le rapport de masculinité est plus élevé chez les immigrants que chez les natifs. Les groupes d'âge sont à peu près également représentés, sauf le groupe d'âge des 55-64 qui représente seulement $18 \%$ de la population de travailleurs, ce qui reflète plus la diminution de l'activité à cet âge que la taille de la cohorte. Les immigrants sont proportionnellement plus nombreux dans le groupe d'âge 35-44 ans et les natifs plus nombreux dans le groupe d'âge le plus jeune. Les trois grandes métropoles que sont Toronto, Montréal et Vancouver regroupent $36 \%$ de la population à l'étude et près de la moitié de celle-ci habite dans une autre grande ville (RMR et AR). Toutefois, la répartition spatiale des natifs et des immigrants diffère énormément. Tel qu'attendu, les immigrants sont très concentrés dans les grandes régions métropolitaines, en particulier Toronto, et très peu représentés dans les régions non métropolitaines ( Ailleurs au Canada»). On doit signaler que la sélection de notre échantillon (25-64 ans avec diplôme d'études secondaires ou plus) met en exergue cette concentration. Il est d'ailleurs intéressant de noter que la population torontoise âgée de 25 à 64 ans en emploi et détenant au moins un diplôme d'études secondaires est composée davantage d'immigrants (56\%) que de personnes nées au Canada.

Un peu plus du quart $(27,5 \%)$ de la population à l'étude a étudié dans un domaine identifié dans la littérature comme étant plus à risque de surqualification. De l'autre côté, 43,3 \% des individus sont issus de domaines d'études les moins à risque d'inadéquation études-emploi. Finalement, on retrouve 29,2 \% dans la catégorie résiduelle. Comparés aux natifs, les immigrants sont un peu plus représentés à la fois dans les domaines d'études à haut risque de surqualification et à faible risque de surqualification.

Environ trois répondants sur cinq de la population à l'étude atteignent le niveau de littératie (niveau 3) considéré comme étant le niveau minimal nécessaire pour bien fonctionner dans la société moderne. En phase avec la littérature, on remarque des différences importantes entre les immigrants et les natifs. De fait, seulement 43,2 \% des immigrants atteignent le niveau 3 de littératie comparativement à une proportion de $64,4 \% \mathrm{chez}$ les natifs. Les trois quarts $(76,1 \%)$ de l'échantillon ont l'anglais ou le français, les deux langues officielles au Canada, comme langue maternelle. Une fois de plus, des différences importantes sont visibles entre natifs et immigrants. Près de $95 \%$ des natifs déclarent une langue officielle comme langue maternelle, mais ce n'est le cas que de $19 \%$ des immigrants. La moitié des immigrants n'ont ni le français ni l'anglais comme langue maternelle et utilisent une langue non officielle à la maison, les autres $(31,1 \%)$ utilisent une langue officielle à la maison.

\section{ANALYSES MULTIVARIÉES}

Le tableau 2 présente les résultats de régressions logistiques où la variable dépendante prend la valeur de 1 si l'emploi occupé nécessite un diplôme moins élevé que celui détenu, c'est-à-dire que le travailleur est surqualifié par rapport à son emploi. Les résultats sont présentés sous la forme de rapports de cotes $(\mathrm{RC})$ et s'interprètent de la manière usuelle. Par rapport à la catégorie de référence, une catégorie ayant un rapport de cote supérieur à 1 indique que celle-ci est positivement associée au le risque d'être surqualifié et inversement un rapport de cote inférieur à 1 indique un risque moins élevé. 
TABLEAU 2

Modèle logit expliquant la probabilité de surqualification chez les travailleurs de 25 à 64 ans détenant au moins un diplôme d'études secondaires, 2012

\begin{tabular}{|c|c|c|c|c|c|c|}
\hline \multirow{2}{*}{ Variables } & & \multicolumn{5}{|c|}{ Population totale } \\
\hline & & Modèle o & Modèle 1 & Modèle 2 & Modèle 3 & Modèle 4 \\
\hline & & \multicolumn{5}{|c|}{ Rapports de cotes } \\
\hline \multicolumn{2}{|c|}{ Variable d'intérêt analytique } & & & & & \\
\hline \multirow{12}{*}{$\begin{array}{l}\text { Variable } \\
\text { composite des } \\
\text { caractéristiques } \\
\text { des immigrants }\end{array}$} & Natifs (Cat. Réf.) & & & & & \\
\hline & Imm. récents & $5,27^{\star}$ & $5,53^{\star}$ & $3,78^{*}$ & $4,74^{*}$ & $3,37^{\star}$ \\
\hline & Imm. arrivés avant l'âge de 15 ans & 1,07 & 1,12 & 0,97 & 1,08 & 0,95 \\
\hline & Imm. nés dans un pays développé & 0,96 & 0,97 & 0,86 & 0,94 & 0,84 \\
\hline & $\begin{array}{l}\text { Imm. nés en Europe de l’Est/ } \\
\text { Europe centrale }\end{array}$ & $2,37^{\star}$ & $2,60^{*}$ & 1,67 & 2,30 * & 1,54 \\
\hline & Imm. nés aux Philippines & $6,44^{*}$ & $7,66^{*}$ & $5,23^{*}$ & $6,12^{*}$ & $4,35^{*}$ \\
\hline & $\begin{array}{l}\text { Imm. nés dans un pays arabe et } \\
\text { diplômés d'un pays développé }\end{array}$ & 1,21 & 1,38 & 1,04 & 1,21 & 0,93 \\
\hline & $\begin{array}{l}\text { Imm. nés dans un pays arabe et } \\
\text { diplômés d'un autre pays }\end{array}$ & $2,68^{*}$ & $3,07^{\star}$ & $2,16^{\star}$ & 2,50 * & 1,83 \\
\hline & $\begin{array}{l}\text { Imm. nés en Asie et diplômés } \\
\text { d'un pays développé }\end{array}$ & $1,68^{*}$ & $1,83^{*}$ & 1,15 & 1,65 & 1,08 \\
\hline & $\begin{array}{l}\text { Imm. nés en Asie et diplômés } \\
\text { d'un autre pays }\end{array}$ & $2,90^{*}$ & $3,39^{*}$ & $2,13^{*}$ & $2,77^{\star}$ & $1,82^{\star}$ \\
\hline & $\begin{array}{l}\text { Imm. nés ailleurs (Afr., Am. lat., } \\
\text { Océanie) et diplômés d'un pays } \\
\text { développé }\end{array}$ & 1,20 & 1,35 & 1,15 & 1,19 & 1,04 \\
\hline & $\begin{array}{l}\text { Imm. nés ailleurs (Afr., Am. lat., } \\
\text { Océanie) et diplômés d'un autre pays }\end{array}$ & $2,26^{\star}$ & $2,52^{\star}$ & $1,90^{*}$ & $2,18^{*}$ & $1,69^{*}$ \\
\hline \multicolumn{2}{|c|}{ Variables de contrôle } & & & & & \\
\hline \multirow{2}{*}{ Sexe } & Hommes (Cat. Réf.) & & & & & \\
\hline & Femmes & & $1,42^{*}$ & $1,43^{*}$ & $1,41^{*}$ & $1,42^{*}$ \\
\hline \multirow{4}{*}{ Groupe d'âge } & 25-34 ans (Cat. Réf.) & & & & & \\
\hline & $35-44$ ans & & $0,74^{\star}$ & $0,74^{\star}$ & $0,74^{*}$ & $0,74^{\star}$ \\
\hline & $45-54$ ans & & $0,77^{\star}$ & $0,77^{\star}$ & $0,73^{*}$ & $0,74^{\star}$ \\
\hline & $55-64$ ans & & $0,78^{*}$ & $0,79^{*}$ & $0,72^{*}$ & $0,73^{*}$ \\
\hline
\end{tabular}


TABLEAU 2 (suite)

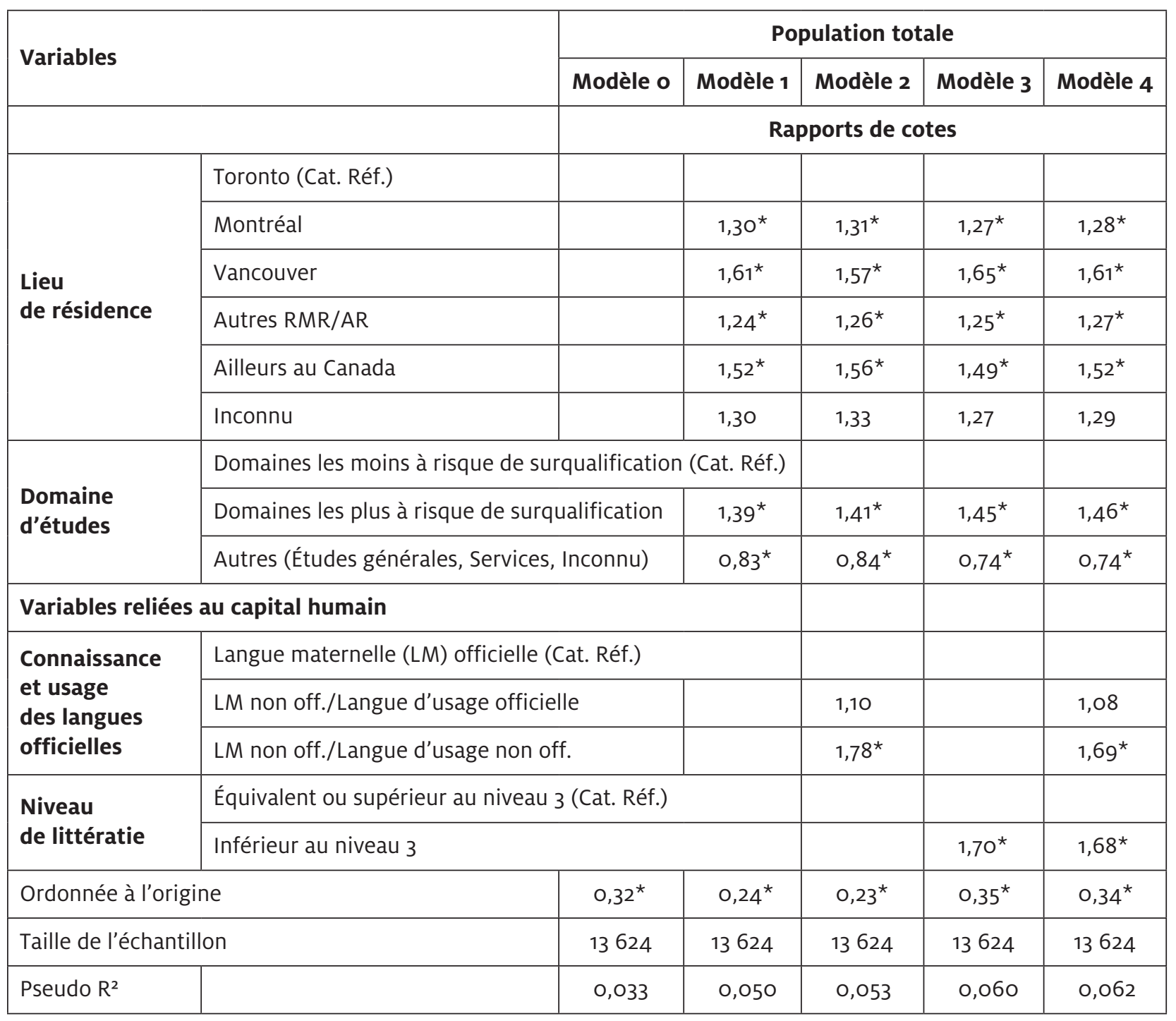

Source: PEICA, 2012.

Le modèle o présente l'effet brut de la variable statut d'immigrants, celle-ci étant la variable d'intérêt dans cette étude. On retrouve ici essentiellement les mêmes résultats que lorsque l'on compare de façon descriptive les taux de surqualification des différents groupes (voir Ledent et ses collaborateurs (2014) pour une démonstration). Les modèles 1 à 4 présentent les effets nets de chacune de variables incluses dans le modèle. Le modèle 1 comprend les variables de contrôle, alors qu'on ajoute respectivement aux modèles 2 et 3 la dimension des compétences linguistiques et des compétences en littératie. Finalement, le modèle 4, le modèle complet, comprend à la fois la variable d'intérêt analytique, les variables de contrôle et les variables mesurant les compétences linguistiques et de littératie. Les rapports de cotes marqués d'un astérisque ${ }^{*}$ ) sont statistiquement significatifs au seuil de $95 \%$. 
Dans le modèle sans contrôle (modèle o), on observe que pratiquement toutes les catégories de la variable composite des caractéristiques des immigrants présentent un rapport de cote supérieur à 1,00 et que la plupart sont significatifs. Par exemple, lorsqu'on ne neutralise pas l'effet des autres caractéristiques, les immigrants récents présentent un risque 5,27 fois plus élevé que les natifs d'occuper un emploi pour lequel ils sont surqualifiés. Ce risque est encore plus élevé pour les immigrants originaires des Philippines $(\mathrm{RC}=6,44)$. Les Européens de l'est et du centre ainsi que les immigrants originaires de pays moins développés affichent un risque de surqualification plus de deux fois plus élevé que celui des natifs et celui-ci est plus élevé lorsque leur diplôme a été obtenu dans un pays moins développé. Ces rapports de cotes élevés sont le reflet du taux de surqualification élevé observé chez les travailleurs nés à l'étranger lorsque l'on ne tient pas compte des effets de structure. Toutefois, même dans le modèle sans contrôle, les immigrants admis avant l'âge de 15 ans (génération 1,5) et ceux originaires d'un pays développé ne se distinguent pas statistiquement des natifs quant à leur chance d'occuper un emploi pour lequel ils seraient surqualifiés. Également, comparativement aux Canadiens nés au pays, le risque de surqualification n'est pas significativement plus élevé pour deux catégories d'immigrants : ceux nés dans un pays arabe et diplômés d'un pays développé ainsi que ceux originaires d'Afrique, d'Amérique latine et de l'Océanie et diplômés d'un pays développé.

Selon le modèle 1 , on voit que toutes les variables de contrôle sociodémographiques sont statistiquement significatives. Les femmes et les jeunes ont plus de chance d'être surqualifiés dans l'emploi qu'ils occupent. Par rapport aux résidents de la région métropolitaine de Toronto, à caractéristiques égales, les résidents des autres régions sont plus susceptibles de connaitre la surqualification et le regroupement des domaines d'études présente les effets attendus. Il est intéressant de noter que les rapports de cotes des catégories de la variable composite du statut d'immigrant qui sont significatifs au modèle o le demeurent lorsqu'on contrôle pour les caractéristiques sociodémographiques et que les rapports de cotes sont sensiblement plus élevés dans le modèle 1 que dans le modèle o. Cela indique que les immigrants ont généralement des caractéristiques sociodémographiques favorables à un faible risque de surqualification.

Les modèles 2 et 3 ajoutent séparément la variable de compétences linguistiques et celle de la compétence en littératie, respectivement. Tel qu'attendu, les travailleurs dont la langue maternelle n'est pas une des deux langues officielles présentent des rapports de cotes plus élevés que ceux de langue maternelle anglaise ou française. Cela est particulièrement vrai pour les allophones qui utilisent une langue autre que le français ou l'anglais le plus souvent à la maison, ceux-ci présentant un risque d'être surqualifié dans l'emploi qu'ils occupent qui est $78 \%$ plus élevé que celui des travailleurs de langue maternelle officielle $(\mathrm{RC}=1,78)$. Par contre, on doit noter que pour les allophones qui utilisent une langue officielle à la maison, bien que le rapport de cote atteigne 1,10, celui-ci n'est pas statistiquement différent de celui du groupe de référence. On peut donc penser que la langue d'usage est un meilleur indicateur de l'intégration économique des immigrants que la langue maternelle. Le fait de prendre en compte la dimension des compétences linguistiques dans le modèle d'analyse n'a pas d'impact sur les rapports de cote des variables de contrôle, mais fait baisser la valeur des rapports de cote de toutes les catégories de la variable statut d'immigrant qui étaient positivement et significativement associés au risque d'être surqualifié. De plus pour deux de ces catégories, soit pour les Européens de l'est et du centre et pour les Asiatiques détenant un diplôme d'un pays 
développé, le rapport de cote n'est plus significativement différent de celui des natifs. Autrement dit, à compétences linguistiques égales, nos résultats suggèrent que ces deux sous-groupes d'immigrants ne sont pas statistiquement plus à risque de surqualification que les Canadiens nés au pays.

Tel qu'attendu, au modèle 3, les compétences en littératie des individus ont un impact significatif sur le risque de surqualification. En effet, le fait d'avoir des compétences en littératie inférieures au niveau 3 augmente significativement et de manière importante $(R C=1,70)$ les risques d'être surqualifié pour l'emploi occupé. Tout comme l'ajout des compétences linguistiques, l'ajout des compétences en littératie dans le modèle d'analyse ne modifie pas substantiellement les rapports de cote des variables sociodémographiques (variables de contrôle). Par contre, tout comme la maîtrise des langues officielles, le fait d'avoir des compétences minimales en littératie permet d'expliquer une bonne partie des écarts de surqualification observés entre les immigrants et les natifs (modèle o). Les rapports de cote de toutes les catégories de la variable statut d'immigrant qui sont significatifs et supérieurs à l'unité diminuent de manière importante, mais moins que dans le cas de l'ajout de la variable linguistique. Par contre, on notera que le pseudo $\mathrm{R}^{2}$ du modèle 3 est légèrement plus élevé que celui du modèle 2, indiquant que la variable mesurant la compétence en littératie possède un pouvoir explicatif plus grand que la variable linguistique. Cela suggère que la variable de littératie est un meilleur indicateur que la variable linguistique pour déterminer le risque de surqualification des travailleurs canadiens. De fait, la quasi-totalité des natifs a une des deux langues officielles comme langue maternelle, alors que le niveau de littératie varie plus fortement.

Le modèle 4 montre que l'insertion conjointe des variables linguistiques et de compétences en littératie ajoute au pouvoir explicatif du modèle sans ajouter de colinéarité indue. En effet, on observe que les rapports de cotes des deux variables demeurent significatifs et leurs valeurs sont très proches de celles des modèles où chacune des variables est incluse séparément.

Finalement, on note dans le modèle complet (modèle 4) que seules quatre catégories de la variable composite présentent des rapports de cote supérieurs à l'unité tout en demeurant significatifs. Les immigrants admis depuis moins de cinq ans et les immigrants originaires des Philippines sont trois à quatre fois plus susceptibles que les natifs d'occuper un emploi pour lequel ils sont surqualifiés. Les immigrants d'origine asiatique ou d'origine africaine ou latino-américaine ou autre, mais qui dans ces deux cas ont obtenu leur plus haut diplôme d'une institution d'un pays moins développé sont aussi plus susceptibles que les Canadiens de naissance d'être surqualifiés. Ceci étant, le risque de ces derniers est beaucoup moins élevé que dans le cas des Philippins ou des immigrants récents, leurs rapports de cote atteignant 1,82 et 1,69 respectivement pour les Asiatiques et pour le groupe résiduel des Africains, Latino-américains et autres.

\section{DISCUSSION}

Cette étude ajoute à la compréhension du processus d’intégration économique des immigrants au Canada en mesurant, pour la première fois, l'impact du niveau de littératie sur la propension à occuper un emploi pour lequel le travailleur est surqualifié.

Les résultats montrent que les allophones qui utilisent une des deux langues officielles à la maison et qui ont un niveau de compétences en littératie plus élevé réduisent leurs risques de surqualification, et ce, peu importe leur origine ou le pays d'obtention de leur diplôme. 
Les analyses multivariées montrent qu'en neutralisant l'effet des caractéristiques sociodémographiques et des variables de capital humain, le risque plus élevé de surqualification des immigrants diminue de $13 \%$ à $46 \%$ selon le type d'immigrant ${ }^{9}$. Les catégories de la variable composite pour lesquelles la diminution est la plus faible sont celles où le risque de surqualification n'est pas significativement différent de celui des natifs. Si on ne considère que les catégories présentant des rapports significatifs, le risque de surqualification décroît entre $33 \%$ et $46 \%$. Les catégories d'immigrants qui présentent la plus forte diminution relative de leur rapport de cote entre le modèle complet (4) et le modèle 1 (avec variables de contrôle) sont, dans l'ordre : les immigrants d'origine asiatique détenant un diplôme d'un pays non développé (-46\%), ceux originaires des Philippines $(-43 \%)$, ceux originaires de l'Europe de l'Est $(-41 \%)$, ceux d'origine asiatique diplômés d'un pays développé $(-41 \%)$, ceux originaires d'un pays arabe diplômés d'un pays non développé $(-40 \%)$ et les immigrants récents $(-39 \%)$. Pour tous ces groupes d'immigrants, les résultats permettent de conclure que des compétences moindres en littératie et une moins grande maitrise des langues officielles expliqueraient grosso modo $40 \%$ des différences de risque de surqualification avec les Canadiens nés au pays.

De plus, pour de nombreuses catégories d'immigrants, les résultats ne montrent aucune différence significative par rapport aux Canadiens de naissance lorsqu'on neutralise l'effet des compétences linguistiques et de littératie. C'est le cas des immigrants originaires d'Europe de l'Est et d'Europe centrale, des immigrants d'origine arabe détenant un diplôme d'une institution « d'un autre pays ${ }^{10}$ » et des immigrants d'origine asiatique détenant un diplôme provenant d'un pays développé. Pour ces catégories d'immigrants, la forte surqualification observée dans le modèle 1 est en fait dû à un effet de structure. En d'autres termes, la plus grande surqualification de ces immigrants vis-à-vis leurs homologues nés au Canada est une observation trompeuse. De fait, à compétences égales, le risque de ces immigrants à occuper un emploi surqualifié n'est pas plus grand que celui des personnes nées au Canada. Autrement dit, nos résultats suggèrent que ces immigrants sont globalement plus surqualifiés que les natifs du fait de leur faible niveau de littératie et de maîtrise des langues officielles.

Toutefois, les compétences linguistiques et en littératie n'expliquent pas tout. Des différences significatives persistent pour certains groupes d'immigrants par rapport aux natifs quant au risque d'être surqualifié, et ce, même lorsque l'on contrôle l'effet de ces variables. Les immigrants récents $(\mathrm{RC}=3,37)$ et les immigrants originaires des Philippines $(\mathrm{RC}=4,35)$ sont particulièrement plus susceptibles d'être surqualifiés que les natifs. De la même manière que la surqualification est plus élevée chez les jeunes en début de carrière, les immigrants récents peuvent connaitre un épisode de surqualification au cours d'un processus naturel d'accès à un emploi qualifié. Il ne faut peut-être pas s'en inquiéter si cette situation demeure temporaire. Par contre, le programme des aides familiaux du Canada (PAFC) par lequel la majorité des Philippins, en particulier les femmes, accède au statut de résident permanent semble les confiner à des emplois peu qualifiés, malgré leur niveau de scolarité relativement élevé. Dans une moindre mesure, les immigrants diplômés d'un pays en développement originaires d'Asie $(\mathrm{RC}=1,82)$ ou d'une autre région (Afrique, Amérique latine ou

9. (RC du modèle 1 - RC du modèle 4)/RC du modèle 1 , en pourcentage.

10. Par opposition aux pays développés. 
Océanie) ( $R C=1,69)$ sont aussi plus susceptibles d'être surqualifiés pour l'emploi qu'ils occupent, alors que ceux originaires des mêmes régions, mais qui sont diplômés d'un pays développé, ne le sont pas lorsque l'on contrôle pour les compétences. Ces résultats tendent à supporter les conclusions de Sweetman $(2004,2014)$ en ce qui concerne la différence de qualité de l'éducation et la transférabilité internationale du capital humain. Ils tendent aussi à infirmer l'hypothèse de la discrimination. En effet, si les immigrants originaires d'une même région connaissent plus de surqualification seulement s'ils ne sont pas diplômés d'un pays développé, c'est donc que l'origine ethnoculturelle n'est pas la cause de celle-ci.

\section{CONCLUSION}

Cette étude montre que le niveau de littératie, tel que mesuré par l'enquête PEICA, permet d'expliquer entre $30 \%$ et $46 \%$ de la surqualification accrue de certaines catégories d'immigrants les plus affectés par le phénomène. D’autres études ont montré des relations similaires, voire plus importantes, entre le niveau de littératie et les différences de revenu (Bonikowska et collab. 2008; Ferrer et collab. 2006; Green et Riddell, 2013; Kahn, 2004).

Depuis le début des années 1990, peu importe la conjoncture économique ou les variations du niveau de chômage, les niveaux d'immigration canadiens demeurent élevés, tant en comparaison avec les niveaux antérieurs qu'en comparaison avec les taux d'immigration observés dans la plupart des autres pays occidentaux, y compris les États-Unis. Pour Green et Green (2004), cela démontre que la politique d'immigration a pour objectif davantage la construction de l'État (nation building) qu'un plan précis pour faire prospérer l'économie du pays et le bien-être de ses habitants.

Pourtant, tout un argumentaire économique est plus souvent mis de l'avant par le gouvernement pour justifier et motiver la mise en place de cibles élevées en matière d'immigration. Depuis plus de 25 ans, on met de l'avant l'immigration comme la solution tous azimuts contre l'inéluctable vieillissement démographique, comme la solution à l'éventuelle pénurie de main-d'œuvre qui en découlerait, ou encore pour pallier une croissance économique défaillante. Pourtant, on discute bien plus souvent du nombre d'immigrants qui devraient être admis chaque année que de leur intégration. Dans un contexte où les immigrants représentent une part importante et croissante de la main-d'œuvre ${ }^{11}$, il est essentiel de favoriser une intégration économique pleine et entière des nouveaux arrivants. Une intégration économique réussie des nouveaux immigrants exige de meilleurs critères de sélection et des investissements en formation pour assurer la mise à niveau des compétences des immigrants.

La grille de sélection accorde un maximum de 25 points pour le niveau d'éducation (Citoyenneté et immigration Canada, 2015). Un même niveau d'éducation entraine le même nombre de points, peu importe le domaine d'étude ou lieu d'obtention du diplôme. Pourtant, les études montrent que la qualité peut varier selon l'origine (Sweetman, 2004) et que le capital humain d'un

11. Selon les projections, les immigrants pourraient représenter jusqu'à $31 \%$ de la population active du Canada (Bélanger et Bastien, 2013; Martel et collab. 2011). 
immigrant n'est pas nécessairement transférable du pays d'origine au pays d'accueil (Chiswick et Miller, 2009b; Sweetman, 2014).

Avant 2012, le nombre de points accordés pour la connaissance des langues officielles était basé sur une évaluation subjective au cours d'un entretien. Un candidat à l'immigration pouvait obtenir le nombre maximal de points sans avoir une excellente connaissance du français ou de l'anglais, surtout lorsque les niveaux d'immigration augmentaient (Dubreuil et Marois, 2011). Depuis, les compétences linguistiques des immigrants sont mesurées à l'aide de tests objectifs. On peut souhaiter que cela favorise une meilleure intégration économique de ceux-ci.

De la même manière, on pourrait mesurer objectivement les compétences des immigrants sélectionnés en vertu du volet économique de la politique d'immigration plutôt que d'accorder des points selon le niveau d'éducation sans égard au domaine d'étude ou au lieu d'obtention du diplôme. S'il est possible de faire une enquête internationale pour mesurer de manière objective les compétences des individus, il est certainement possible d'utiliser des outils similaires pour mesurer les compétences en littératie des candidats à l'immigration. Certes, cela entrainera des coûts, mais puisque l'immigration est appelée à représenter une part croissante de la maind'œuvre, il est essentiel de s'assurer que les critères de sélection des immigrants de la catégorie économique permettent d'accroître les chances que ceux-ci puissent s'insérer avec succès sur le marché du travail canadien.

\section{BIBLIOGRAPHIE}

AYDEMIR, A. et M. SKUTERUD. 2004. Explaining the Deteriorating Entry Earnings of Canada's Immigrant Cohorts : 1966-200o. Ottawa, Statistics Canada, 32 p. ( $\mathrm{n}^{\circ} 11$ Foo19MIE - No. 225 au catalogue).

BÉLANGER, A. et N. BASTIEN. 2013. "Immigration, Education, Ethnocultural Diversity and the Future Composition of the Canadian Labour Force », Population and Development Review, 39, $3: 509-525$.

BÉRARD-CHAGNON, J. 2015. Les compétences en littératie des francophones de l'Ontario : état des lieux et enjeux émergents. Ontario, Emploi Ontario, 199 p.

BONIKOWSKA, A., A. G. GREEN et W. C. RIDDELL. 2010. "Immigrant Skills and Immigrant Outcomes under a Selection System : The Canadian Experience », Conference on the Economics of Immigration, 2010, Ottawa.

BONIKOWSKA, A., D. A. GREEN et W. C. RIDDELL. 2008. Littératie et marché du travail : les capacités cognitives et les gains des immigrants. Ottawa, Statistique Canada, 73 p. $\left(\mathrm{n}^{\circ}{ }_{11 \mathrm{Foo}} \mathrm{M}-\right.$ No. 330 au catalogue).

BOUDARBAT, B. et C. MONTMARQUETTE. 2013. Origine et sources de la surqualification dans la région métropolitaine de Montréal. Montréal, CIRANO, 116 p. (Rapport de projet n²013RP-08).

CARON MALENFANT, É., A. LEBEL et L. MARTEL. 2010. Projections de la diversité de la population canadienne. Ottawa, Statistique Canada, 82 p. ( $\mathrm{n}^{\circ}$ 91-551-X au catalogue).

CAUSA, O. et S. JEAN. 2007. Integration of Immigrants in OECD Countries : Do Policies Matter?. Paris, OECD Publishing, 34p. (Economics Department Working Papers n 564 ). 
CHISWICK, B. R. 1978. "The Effect of Americanization on the Earnings of Foreign-born Men », Journal of Political Economy, 86, 5 : 897-921.

CHISWICK, B. R. et P. W. MILLER. 2002. "Immigrant earnings : Language skills, linguistic concentrations and the business cycle », Journal of population economics, 15, 1: 31-57.

- 2009a. An explanation for the lower payoff to schooling for immigrants in the Canadian labour market. Bonn, IZA, 43 p. (Institute for the Study of Labor Discussion Paper $n^{\circ} 4448$ ).

- 2009 b. "The international transferability of immigrants' human capital ", Economics of Education Review, 28 : 162-169.

CITOYENNETÉ ET IMMIGRATION CANADA. 2015. Les six critères de sélection - Travailleurs qualifiés (fédéral). Consulté le 18 août 2016. http ://www.cic.gc.ca/francais/immigrer/qualifie/ demande-facteurs.asp.

CORBEIL, J.-P. 2011. "Acquis et défis de la mesure statistique des niveaux de littératie des immigrants de tierce langue maternelle », Cahiers de l'Institut des langues officielles et du bilinguisme (ILOB), $3: 31-48$.

DUBREUIL, B. et G. MAROIS. 2011. Le Remède imaginaire: Pourquoi l'immigration ne sauvera pas le Québec. Montréal, Boréal, 320 p.

DUMONT, J.-C. et O. MONSO. 2007. "Adéquation entre formation et emploi : un défi pour les immigrés et les pays d'accueil », dans OCDE (dir.), Perspectives des migrations internationales. Paris, SOPEMI (Système d'observation permanente des migrations) : 141-170.

FERRER, A., D. A. GREEN et W. C. RIDDELL. 2006. "The Effect of Literacy on Immigrant Earnings », The Journal of Human Resources, 41, $2: 380-410$.

FERRER, A. et W. C. RIDDELL. 2004. "Education, credentials, and immigrant earnings ", Canadian Journal of Economics, 41, 1:186-216.

FRENETTE, M. et R. MORISSETTE. 2005. «Will they ever converge ? Earnings of immigrant and Canadian-born workers over the last two decades ", International Migration Review, 39, 1 : 228-258.

GALARNEAU, D. et R. MORISSETTE. 2004. "Les immigrants sont-ils perdants? ", Perspective, 5, $6: 5$-17. ( ${ }^{\circ} 75$-001-XIF au catalogue de Statistique Canada).

- 2008. "Scolarité des immigrants et compétences professionnelles requises. ", Perspective, 9, 12 : 5-19. ( $\mathrm{n}^{\circ}$ 75-001-XIF au catalogue de Statistique Canada).

GREEN, A. G. et D. A. GREEN. 2004. «The goals of Canada's immigration policy : A historical perspective », Canadian Journal of Urban Research, 13, 1:102-139.

GREEN, C., P. KLER et G. LEEVES. 2007. «Immigrant overeducation : Evidence from recent arrivals to Australia ", Economics of Education Review, 26, 4:420-432.

GREEN, D. A. et W. C. RIDDELL. 2013. "Ageing and literacy skills : Evidence from Canada, Norway and the United States ", Labour Economics, 22 : 16-29.

GREEN, D. A. et C. WORSWICK. 2012. "Immigrant earnings profiles in the presence of human capital investment: Measuring cohort and macro effects", Labour Economics, 19, 2 : 241-259.

HARTOG, J.. 2000. "Over-education and earnings : where are we, where should we go ? ", Economics of Education Review, 19, $2:$ 131-147.

HOU, F. et G. PICOT. 2014. Niveaux annuels d'immigration et gains initiaux des immigrants au Canada. Ottawa, Statistics Canada, 39 p. (n 11 Foo19M - No. 356 au catalogue). 
HUM, D. et W. SIMPSON. 2000. "Not all visible minorities face labour market discrimination », Policy Options 21, $10: 45-48$.

KAHN, L. M. 2004. "Immigration, skills and the labor market : International evidence », Journal of population economics, $17:$ 501-534.

LAROCHELLE-CÔTÉ, S. et D. HANGO. 2016. La surqualification, les compétences et la satisfaction au travail. Ottawa, Statistics Canada, 19 p. ( $\mathrm{n}^{\circ}$ 75-006-X au catalogue).

LEDENT, J., A. BÉLANGER et G. MAROIS. 2014. La surqualification des salariés d'origine immigrée résidant sur l'île de Montréal en 2006. Montréal, Institut national de la recherche scientifique, Centre - Urbanisation Culture Société, 77 p.

LI, P. S. 2003. " Initial Earnings and Catch-up Capacity of Immigrants », Canadian Public Policy/ Analyse de Politiques, 29, $3:$ 319-337.

LUBOTSKY, D. 2007. "Chutes or Ladders? A Longitudinal Analysis of Immigrant Earnings », Journal of Political Economy, 115, $5: 820-867$.

MARTEL, L., É. CARON MALENFANT, J.-D. MORENCY, A. LEBEL, A. BÉLANGER et N. BASTIEN. 2011. «La population active canadienne en 2031 : tendances projetées à l'horizon 2031 », L'observateur économique canadien, 24, $8: 3.1-3.13$.

OCDE. 2012. Trouver ses marques - Les indicateurs de l'OCDE sur l'intégration des immigrés 2012. Paris, Éditions OCDE, 165 p.

- 2014. L'évaluation des compétences des adultes : Manuel à l'usage des lecteurs. Paris, Éditions OCDE, $138 \mathrm{p}$.

PENDAKUR, K. et R. PENDAKUR. 1998. "The colour of money : earnings differentials among ethnic groups in Canada ", Canadian Journal of Economics, 31, 3: 518-548.

— . 2002. "Speaking in Tongues : Language Knowledge as Human Capital and Ethnicity ", International Migration Review, 36, 1: 147-178.

PICOT, G. et F. HOU. 2009. The effect of immigrant selection and the it bust on the entry earnings of immigrants. Vancouver, Canadian Labour Market and Skills Researcher Network, 38 p. (Working Paper $n^{\circ} 29$ ).

PICOT, G., F. HOU et S. COULOMBE. 2008. « Poverty dynamics among recent immigrants to Canada. ", International Migration Review, 42, $2:$ 393-424.

POKROPEK, A. et M. JAKUBOWSKI. 2014. PIAACTOOLS : Stata programs for statistical computing using PIAAC data. Paris, OECD Publishing, $15 \mathrm{p}$.

PORTER, J. 1965. The Vertical Mosaic : An Analysis of Class and Power in Canada. Toronto, University of Toronto Press, $626 \mathrm{p}$.

PORTES, A. et M. ZHOU. 1993. "The New Second Generation : Segmented Assimilation and its Variants ", The ANNALS of the American Academy of Political and Social Science, 530, 1: 74-96.

ROY, N. 2014. «La surqualification au Québec et en Ontario : l'évolution comparée de l'emploi hautement qualifié et des personnes hautement scolarisées », dans M. VULTUR (dir.), La surqualification au Québec et au Canada. Québec, Presses de l'Université Laval : 49-72.

SABOURIN, P. et A. BÉLANGER. 2014. "Microsimulation of language characteristics and language choice in multilingual regions with high immigration », dans IMA et Statistics Sweden (dir.), Proceedings of the Sixth Eurostat/UNECE Work Session on Demographic Projections. Rome, Italie : 443-456. 
SCHAAFSMA, J. et A. SWEETMAN. 2001. "Immigrant earnings : age at immigration matters », Canadian Journal of Economics, 34, $4:$ 1066-1099.

SKUTERUD, M. 2011. Language skills in the new economy and the deteriorating labour market performance of Canada's immigrant workers. Waterloo, University of Waterloo, $59 \mathrm{p}$.

STATISTIQUE CANADA. 2013a. Enquête nationale auprès des ménages de 2011 : Tableaux de données. Ottawa, Statistique Canada ( $\mathrm{n}^{\circ}$ 99-012-X2011042 au catalogue)

- 2013 b. Immigration et diversité ethnoculturelle au Canada. Ottawa, Statistique Canada, $24 \mathrm{p}$. ( ${ }^{\circ}$ 99-010-X2011001 au catalogue).

—. 2013c. Indicateurs de l'éducation au Canada : une perspective internationale. Ottawa, Statistique Canada, 136 p. ( $\mathrm{n}^{\circ} 81-604-\mathrm{X}$ au catalogue).

— $2013 \mathrm{~d}$. Les compétences au Canada: Premiers résultats du Programme pour l'évaluation internationale des compétences des adultes (PEICA). Ottawa, Statistique Canada, $117 \mathrm{p} .\left(\mathrm{n}^{\circ} 89-555-\mathrm{X}\right.$ au catalogue).

——. 2015. Glossaire. Consulté le 19 avril 2017. http://www.statcan.gc.ca/pub/92-637-g/ 2011001/2011005-fra.htm.

2016. Estimations démographiques annuelles: Canada, provinces et territoires 2016. Ottawa, Statistique Canada, 192 p. ( $\mathrm{n}^{\circ}$ 91-215-X au catalogue).

STATISTIQUE CANADA et OCDE. 2005. Apprentissage et réussite - Premiers résultats de l'enquête sur la littératie et les compétences des adultes. Ottawa et Paris, Éditions OCDE, 339 p.

SWEETMAN, A. 2004. Qualité de l'éducation des immigrants dans leur pays d'origine et résultats sur le marché du travail canadien. Ottawa, Statistique Canada, 47 p. ( ${ }^{\circ} 11$ Foo19MIF - No. 234 au catalogue).

—_. 2014. «La transférabilité internationale du capital humain des immigrés : L'expérience du Canada ", dans OCDE et Union Européenne (dir.), Gérer les migrations économiques pour mieux répondre aux besoins du marché du travail. Paris, Éditions OCDE : 251-272.

SWEETMAN, A. et S. MCBRIDE. 2004. Domaines d'études postsecondaires et résultats sur le marché du travail canadien des immigrants et des non-immigrants. Ottawa, Statistique Canada, 81 p. ( ${ }^{\circ}$ 11Foo19MIF - No. 233 au catalogue).

UPPAL, S. et S. LAROCHELLE-CÔTÉ. 2014. La surqualification des nouveaux diplômés universitaires au Canada. Ottawa, Statistique Canada, 15 p. ( $\mathrm{n}^{\circ}$ 75-006-X au catalogue). 